\title{
Schlaf und Traum
}

Erhard Taverna

Ein Drittel unseres Lebens geht im Schlaf vorbei, gute Gründe für eine Expedition in die Nachtzonen unserer Existenz. Das Deutsche HygieneMuseum in Dresden zeigt noch bis zum 3. Oktober 2007 in Zusammenarbeit mit der Wellcome Collection London, wie Geschichte und Mythologie, Kunst und Soziologie und nimmermüde Mediziner und Biologen diesen sonderbaren Zustand erkunden. Dabei bleibt wissenschaftlich ungeklärt, warum wir den Schlaf überhaupt brauchen.

Seit Kleitman in Chicago die REM-Phasen entdeckt hat, haben der Schweizer Alexander Borbély und viele andere Forscher das klassische Diagramm der zyklisch wechselnden Schlafperioden weiterentwickelt. Das Gehirn braucht immer Glukose und schläft nie, das weiss man seit der Erfindung des EEG in den 1920er Jahren und den PET-Scans der 80er. Mehrfach wird die Arbeit im Schlaflabor dokumentiert, mit polysomnographischen Aufzeichnungen, Infrarotaufnahmen und Filmsequenzen zu Parasomnien, bis hin zur letalen, familiären Schlafunfähigkeit, einer seltenen Prionenkrankheit. Die Welt der Schnarcher ist ebenso vertreten wie jene der Schlafwandler und Narkoleptiker, die der Schlafund jene der Wachmittelsüchtigen.

Die interdisziplinäre Ausstellung «Schlaf \& Traum» bietet faszinierende Einblicke in die zahlreichen biomedizinischen und kulturellen Wechselwirkungen, die in jeder Epoche zu verschiedenen Resultaten führten. Filme und Modelle, Audioinstallationen, Kunstobjekte, Forschungsinstrumente, Frage-und-Antwort-Spiele und interaktive PC-Stationen bieten zahlreiche Einblicke in das nächstliegende und doch ferne Phänomen. Nyx, die eulenbegleitete Göttin der Nacht, trägt das Zwillingspaar Hypnos und Thanatos auf dem Arm, damit hat auch die Traumforschung ihren festen Platz. Das Sigmund-Freud-Institut in Frankfurt am Main weiss, warum traumatische Erlebnisse unlöschbare Spuren im Gehirn hinterlassen, und der südafrikanische Neurologe und Psychoanalytiker Mark Holms zeigt, dass die inneren Hirnregionen die Träume produzieren.

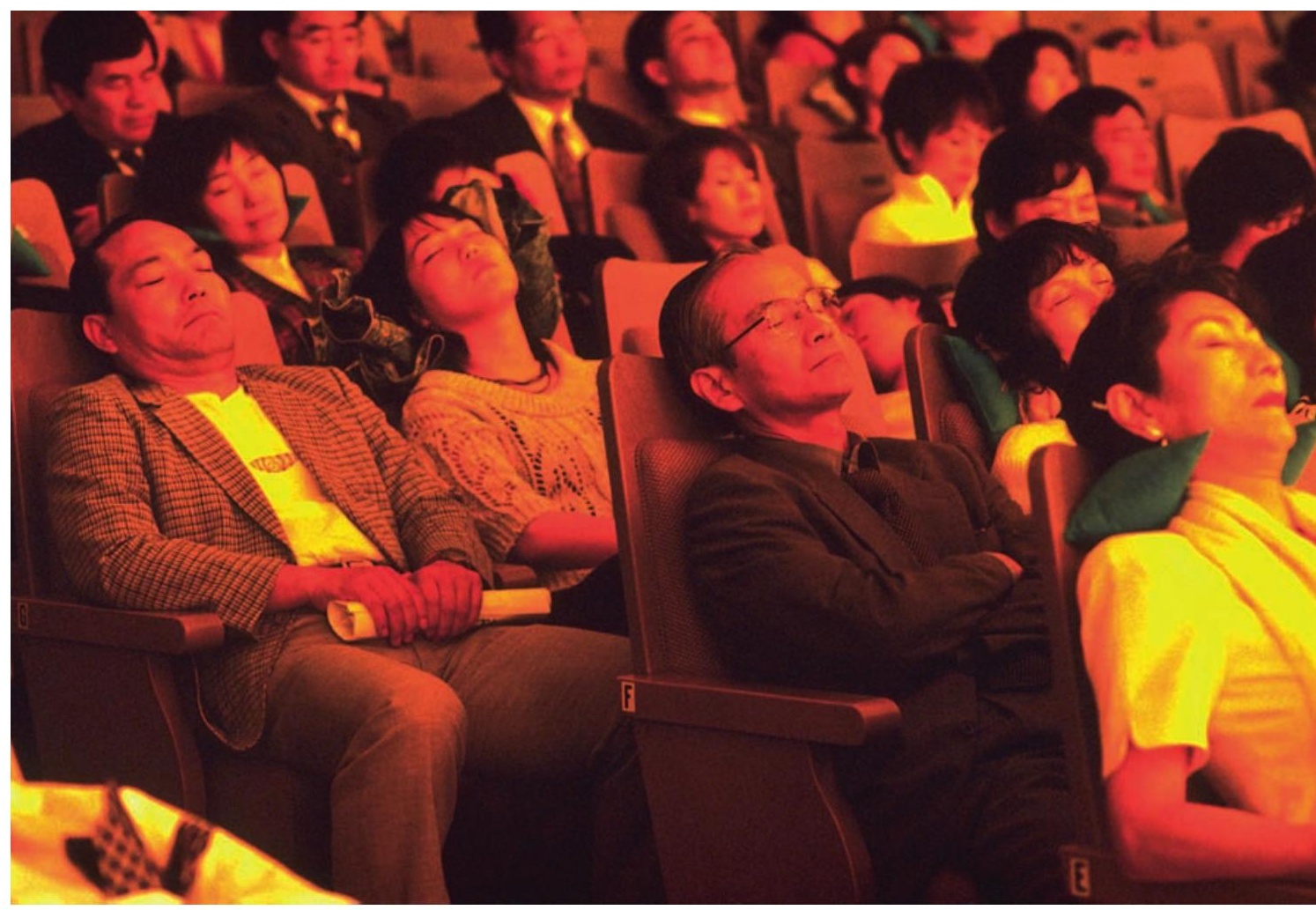

Schlafkonzert von Miyashita Fumio in Japan. @ Nagano (Japan) Biwa Studio. 


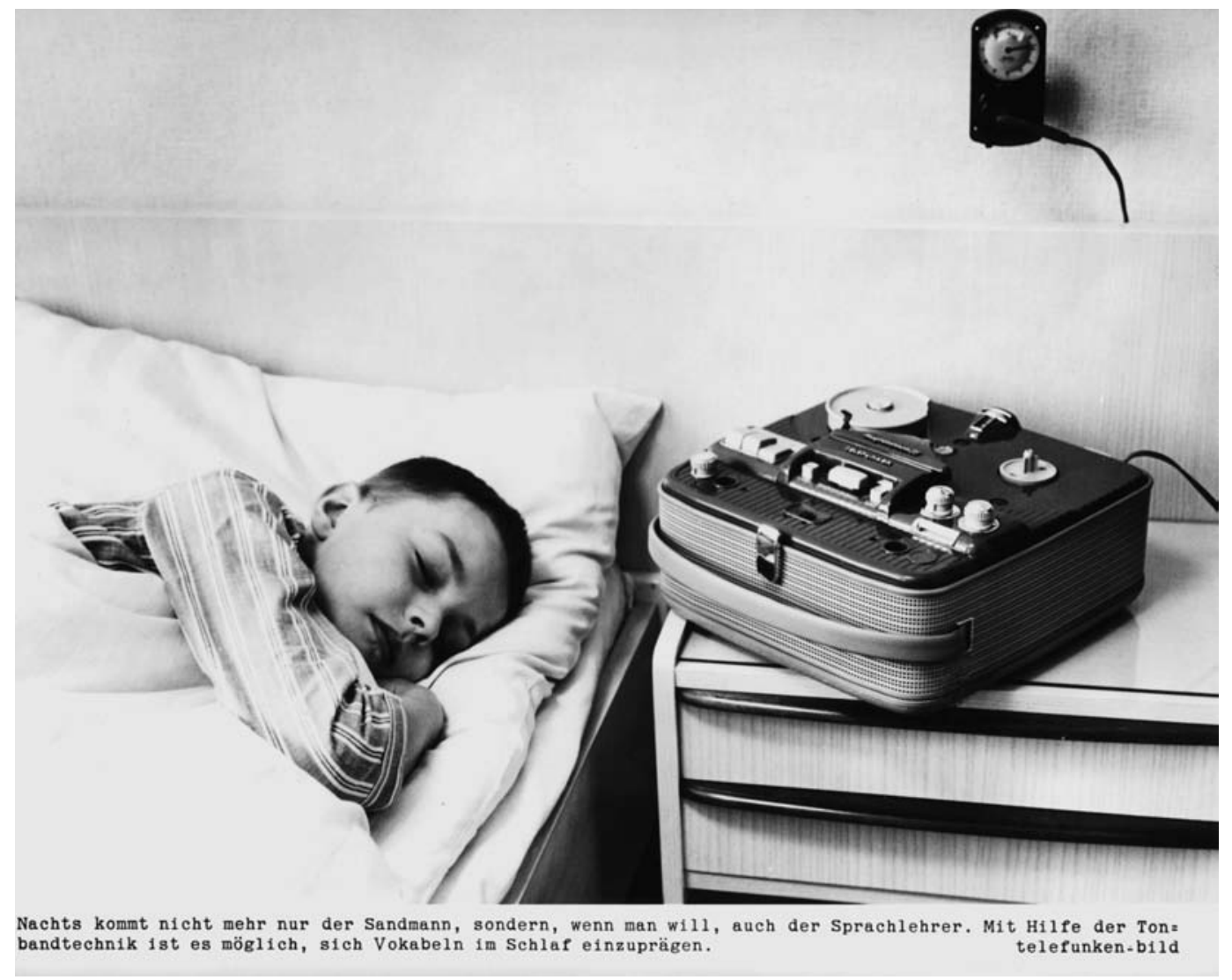

Werbung aus dem Jahr 1963 für das Erlernen von Sprache im Schlaf. Foto: Deutsches Technikmuseum Berlin, Historisches Archiv.

Wer nicht ausreichend schläft, leistet weniger, altert früher und stirbt schneller. Dabei hat jede Kultur eigene Techniken entwickelt, trickreiche Wecker oder eigens komponierte Schlafkonzerte. Es gibt ein phantasievolles Arsenal von Powernappingmöbeln und -accessoires und eine japanische Schlafkunst, die sich Inemuri nennt. Vom Schlafmittel zum Wiegenlied ist alles zu haben. Ein Bett war lange nichts Selbstverständliches, davon zeugen die Schlafgänger der Berliner Unterschicht vom Beginn des letzten Jahrhunderts, Arbeiter, die teils in mehreren Ablösungen ein gemietetes Bett teilten. Photographien von Theo Frey illustrieren die Bettenaktion des Schweizerischen Roten Kreuzes von 1954, die für jedes Kind ein eigenes Bett zum Ziele hatte. Den Seinen gibt's der Herr im Schlaf: Kekulé träumt den Benzolring, Mendelejew sein Periodensystem und Paul McCartney die Melodie von «Yesterday». Alte und vor allem neue Kunst erweitern den Horizont von Schlaf und Traum.

Der Wechsel von Bewusstseins- und Realitätsebenen, die geträumte Aufhebung von Zeit und
Raum, von Kontrolle und Vernunft hat schon immer die Maler, Filmemacher und Dichter inspiriert. Szenen aus Buñuels Film «Un chien andalou» wechseln mit Füsslis «Nachtmahr» und der riesig-schwarzen Polyesterratte von Katharina Fritsch, die mitten in der Ausstellung auf des Schläfers weisser Bettdecke hockt. Die industrialisierte Welt ist voller Licht, in der globalen ist überall Tag und Nacht zugleich. Immer weniger halten sich an die gute alte Mittagszeit, Maschinen und Arbeitsschichten laufen rund um die Uhr, Börsen reagieren in Echtzeit. Hat der Schlaf eine Zukunft, wird er in der beschleunigten Servicewelt kapitulieren, setzt ihm die Glasarchitektur ein Ende? Ist er Zeitverschwendung, ein noch ungenutztes Potential für Lernmanipulationen und weitere arbeitsfördernde Techniken? Die Ausstellung zeigt den Weg zum heutigen Wissen, gibt Antworten und stellt Fragen. Wer die Gelegenheit ergreifen will, besteige den CityNightLiner nach Dresden. Eine lohnende Reise im Schlaf. 